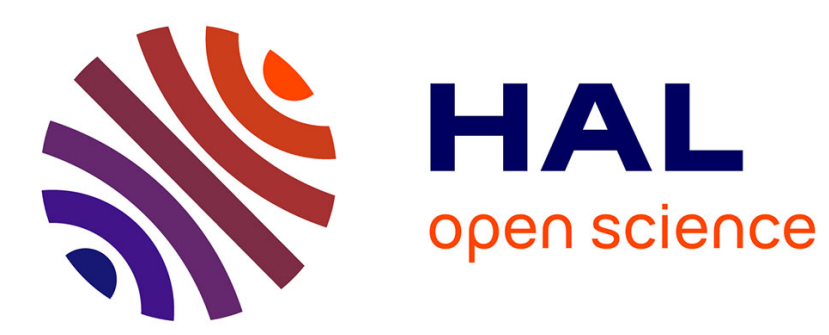

\title{
Semis, transplantation et greffe: les techniques de la compilation dans le Rosarius \\ Marie-Laure Savoye
}

\section{To cite this version:}

Marie-Laure Savoye. Semis, transplantation et greffe: les techniques de la compilation dans le Rosarius. Le livre, le texte, le temps : la mise en recueil au XIIIème siècle, Actes du colloque tenu à Genève les 17 et 18 novembre 2007, Brepols, 2010. halshs-02570835

\section{HAL Id: halshs-02570835 \\ https://shs.hal.science/halshs-02570835}

Submitted on 12 May 2020

HAL is a multi-disciplinary open access archive for the deposit and dissemination of scientific research documents, whether they are published or not. The documents may come from teaching and research institutions in France or abroad, or from public or private research centers.
L'archive ouverte pluridisciplinaire HAL, est destinée au dépôt et à la diffusion de documents scientifiques de niveau recherche, publiés ou non, émanant des établissements d'enseignement et de recherche français ou étrangers, des laboratoires publics ou privés. 


\section{Marie-Laure Savoye}

Institut de Recherche et d'Histoire des Textes

\section{Semis, transplantation et greffe: les techniques de la compilation dans le Rosarius $^{1}$}

Le manuscrit conservé sous la cote fr. 12483 à la Bibliothèque Nationale de France est connu sous le nom de Rosarius depuis la notice textuelle que lui a consacrée Arthur Långfors en 1916². Bien qu'il ait fait l'objet de nombreuses éditions partielles ${ }^{3}$, sa genèse reste encore largement énigmatique. Arthur Långfors éludait lui-même la question qu'il avait posée des rapports de cette compilation « avec le culte du rosaire $»^{4}$. Pour découvrir ce jardin ${ }^{5}$ et tenter de percer le mystère de son agencement, il reste pourtant quelques grilles à ouvrir avec le sésame que nous a fourni le copiste du manuscrit - et sans doute avant lui le compilateur.

Bien malmené par le temps, le codex que nous conservons actuellement se compose de 266 feuillets ou fragments de feuillets (parfois ne reste qu'une mince bande de parchemin, sur laquelle seule la colonne d'initiales est lisible). Il mesure $258 \mathrm{~mm}$ en hauteur et $176 \mathrm{~mm}$ en largeur, mais il a été largement - trop largement - rogné. Sur de très nombreux feuillets, le dernier vers de la colonne est à peine lisible. Au f. 9, la marge de queue avait été utilisée pour copier des vers et le parchemin avait pour cette raison été replié avant rognure du bloc. La bande de parchemin ainsi préservée de la mutilation mesure $37 \mathrm{~mm}$, ce qui

1 Je remercie pour leur disponibilité et leurs précieux conseils Mesdames Gousset et Laffitte, conservateurs du département des manuscrits de la Bibliothèque nationale de France, ainsi que mes collègues de l'Institut de Recherche et d'Histoire des Textes Mesdames Leurquin et Ruby.

2 A. LÅNGFORS, "Notice du manuscrit français 12483 de la Bibliothèque nationale », dans Notices et extraits des manuscrits de la Bibliothèque nationale et autres bibliothèques, 39/2 (1916) 1916, pp. 503-663.

${ }^{3}$ Le manuscrit est connu de longue date, et A. LÅNGFORS, art. cit., signalait déjà plusieurs éditions du $\mathrm{XIX}^{\mathrm{e}} \mathrm{s}$. ou du début du $\mathrm{XX}^{\mathrm{e}}$, éditions portant principalement sur les pièces lyriques. Depuis, M.A. SAVOIE a édité les descriptions commentées de plantes ( $A$ "Plantaire" in honor of the Blessed Virgin Mary taken from a French manuscript of the XIV th century, Washington, Catholic University of America, 1933) ; A. ZETTERBERG et Z. SANDQVIST, celles des «choses» (Les propriétés des choses selon le Rosarius, B.N.F. fr. 12483, Lund, 1994); S. SANDQVIST, celles des animaux et des pierres (Le bestiaire et le lapidaire du Rosarius, Lund, 1996) ; P. KunSTMANN, les miracles (Miracles de Notre-Dame, tirés du Rosarius, Ottawa, 1991).

4 ibid., p. 514.

5 Voir les lignes consacrées à ce manuscrit par G. GROS, «Au jardin des images mariales : aspects du plantaire moralisé dans la poésie religieuse du XIV e siècle », dans Vergers et jardins dans l'univers médiéval, Senefiance 28 (1990), pp. 139-153. 
nous donne par approximation les dimensions de la marge de queue aujourd'hui perdue, et une hauteur restituée de $295 \mathrm{~mm}$. En outre, si l'on fait une hypothèse a minima d'une marge de gouttière équivalente à la marge de couture, soit $16 \mathrm{~mm}$, on aboutit à une page large de $192 \mathrm{~mm}$. En tout état de cause, il devait s'agir d'un volume de dimensions moyennes.

L'évaluation de son épaisseur est plus problématique. Le bloc est actuellement très volumineux, alors que nous déplorons de nombreuses lacunes. Le nombre de vers moyen des chapitres conservés dans leur intégralité est de $785^{6}$. Sur cette base, les deux livres complets du Rosarius, chapitres et prologues, auraient pu compter près de 80000 vers et il nous manquerait quelque 29000 vers, soit 165 feuillets. Ajoutés à ce que nous conservons, cela laisserait supposer un bloc de plus de 430 feuillets, chiffre qui en fait de ce manuscrit sur parchemin un objet codicologique assez exceptionnel ${ }^{7}$

La datation du manuscrit a été controversée. Fort opportunément, il conserve une miniature - une Nativité - au début du second livre, miniature réalisée dans les années 1340 dans un atelier parisien proche de celui de Jeanne et Richard de Montbaston ${ }^{8}$. Ceci conforte l'hypothèse paléographique de Långfors, qui datait l'écriture du « deuxième quart du 14e ». Le terminus post quem pour la composition du recueil textuel est donné par le Dit du Roi de Watriquet de Couvins, dédié à Philippe de Valois (1328-1350), ce qui nous porte après 1328.

L'évocation du Dit du Roi me conduit enfin au vif du sujet, la composition du recueil, puisque d'un recueil il s'agit bien. Le manuscrit est consacré à la Vierge Marie, dont il dit les merveilles en enchevêtrant trois traditions littéraires : les propriétés des choses, les recueils de miracles et les chansonniers.

C'est au niveau microstructural que s'observe cet enchevêtrement, chaque chapitre présentant une architecture assez stable à trois étages :

${ }^{6}$ Ce chiffre a été établi d'après la transcription que j’ai faite de ce manuscrit en vue de son édition intégrale.

7 L'évaluation du volume originel de ce manuscrit reste problématique, d'autant que je montrerai cidessous qu'on me semble fondés à envisager l'existence d'un troisième livre aujourd'hui perdu dans son intégralité. Il faudrait diviser ce bloc de quelque 660 feuillets. Mais le manuscrit ne prévoit pas visiblement de scission aux articulations des livres. En revanche, des lacunes importantes observées pourraient corroborer l'hypothèse d'une répartition des trois livres en quatre volumes : le premier allant jusqu'au chapitre I, 38, le deuxième jusqu'au II, 24 ; le troisième pouvant s'être arrêté vers le chapitre III, 12 (on trouvera dans la notice d'A. LÅNGFORS, art. cit., les précisions nécessaires quant à l'état matériel du manuscrit). Le codex fait actuellement l'objet d'une campagne de restauration qui apportera peut-être quelque nouvel indice.

${ }^{8}$ M.T. GOUSSET reconnaît ici la main d'un artiste proche des Montbaston. Sur l'atelier des Montbaston et ses émules, voir R.H. et M.A. ROUSE, Illiterati et uxorati. Manuscripts and their makers. Commercial Book Producers in Medieval Paris 1200-1500, Londres, Harvey Miller, 2000. 
- une description de «chose» (animal, plante, minéral, mais aussi objet, ou notion), description moralisée, ou plus précisément marialisée ;

- un récit hagiographique (le plus souvent un miracle marial);

- une pièce lyrique ou dévotionnelle (chanson ou prière).

Sur le plan macrostructural, nous conservons les fragments de deux livres : le manuscrit est acéphale, mais on connait le nombre de chapitres du premier livre par la numérotation des rubriques. Au f. 90va, le dernier chapitre porte le numéro L. Le manuscrit est anoure, mais le prologue du second livre est suivi d'une table, annonçant de nouveau cinquante chapitres. C'était là une première clé d'interprétation, bien repérée par Långfors.

La seconde et la plus précieuse vient du repérage marginal qui court tout au long de cette longue copie. Le recueil est en effet constitué de vers composés par le compilateur (exclusivement pour les premier et deuxième étages de l'architecture décrite ci-dessus) et de vers empruntés à d'autres poètes français. Avec une rigueur et une précision qui ne laissent de susciter l'étonnement, le compilateur - ou le relecteur du manuscrit ${ }^{9}$ - balise son texte afin que le lecteur puisse repérer rapidement vers endogènes ou allogènes. En regard des seconds vient un «quid'» parfois développé en «quidem»; devant les premiers un « ros'», une fois seulement « Rosari'» (f. 19v), ce qui permet d'opter pour une résolution latine de l'abréviation en «Rosarius». Bien que cette balise fonctionne en parallèle avec des identifications d'auteurs, c'est bien un titre qu'il faut y voir, et non un pseudonyme. Un détour par une petite histoire des rosaires et autres rosiers permettra de comprendre les motivations complexes de ce recueil marial.

Je résume ici l'histoire d'une dévotion plus polymorphe au Moyen Âge que dans les siècles suivants. La légende en attribue l'invention à saint Dominique ; elle semble plutôt l'aboutissement d'efforts multiples d'enrichissement de la salutation angélique, dont les récits de miracles de la Vierge, toutes langues confondues, disent la place prégnante dans la spiritualité des $\mathrm{XII}^{\mathrm{e}}$ et $\mathrm{XIII}{ }^{\mathrm{e}}$ siècles. L'enchaînement des Ave prend sa source dans ce qu'André Duval

${ }^{9}$ Le manuscrit a fait l'objet d'une relecture plus ou moins attentive contemporaine de sa copie ; certaines des balises marginales sont de la main du relecteur. Il est bien difficile de déterminer s'il s'agit d'une collation sur exemplaire ou d'une série de corrections ou d'annotations d'auteur. La précision du repérage des passages allogènes est telle que seule l'auteur peut en être originellement responsable. Est-ce à dire que nous aurions sous les yeux un manuscrit autographe ? Certains indices peuvent le laisser penser. Peut-on aller jusqu'à lire ce volume comme un brouillon? L'écriture souvent peu soigneuse ainsi que des hésitations entre deux leçons marquées d'un « vel sic» le suggéreraient également, n'était la présence d'une miniature, qui même médiocre, confère au codex le statut tout autre d'œuvre achevée. 
appelle une piété «acclamative et répétitive » ${ }^{10}$. Outre l'accumulation « numérique ", l'enrichissement de la prière passe dès les premiers siècles du christianisme par l'adjonction à la salutation ou à toute autre prière - on pense en particulier au Gaude Maria - de péricopes évangéliques ou de courts textes méditatifs. Un processus comparable vient enrichir le psautier davidique, et des collections de textes se constituent à cette fin. Un rapprochement peut dès lors s'opérer entre une récitation du psautier davidique augmenté de méditations et les séries de prières mariales pareillement enrichies. Dès le XII e siècle sont attestés des psautiers de la Vierge enlaçant prières et méditations. On trouve ainsi mention de Psalteria Mariae chez Césaire d'Heisterbach, Jean de Mailly et surtout Thomas de Cantimpré.

L'analogie avec le psautier davidique structure ces Psalteria en trois séries de cinquante unités, soit un total de cent cinquante prières. Cette somme autorise aussi une autre lecture, l'association de dix Ave Maria à chacune des Quinze Joies de la Vierge. Mais c'est bien la structuration en trois groupes de cinquante qui est la plus fortement perçue. Chacune des cinquantaines reçoit un nom qui peut être corona, sertum, ou rosarium, en français rosaire ou chapelet. Dans ces deux langues - et dans d'autres encore, le terme choisi garde la trace d'une analogie entre la pratique dévotionnelle et la couronne de roses, signe de joie et de fête, offrande à la bien aimée. On connaît le miracle raconté dans les Miracles de Notre-Dame par personnages ${ }^{11}$ (1339-1382), et bien avant dans les Cantigas de Santa Maria ${ }^{12}$, l'histoire d'un chevalier qui avait fait le vœu de tresser tous les jours une couronne de roses à la Vierge, ou à défaut de lui réciter une série d'Ave (le nombre n'en est pas défini) :

E aquesto cada día | podendo-as achar, e senon, por cada rosa | dissesse en seu logar huna vez «Ave Maria », | e assi fosse cercar a guerlanda toda delas, | e que lla fezess' atal.

(Cantigas de Santa María, 121, vv. 10-13)

10 André Duval, art. "Rosaire», dans le Dictionnaire de Spiritualité, t. XIII, col. 937-980, Paris, Beauchesne, 1988. Les lignes qui suivent empruntent largement à cet article, ainsi qu’à M.M. GORCE, art. «Rosaire», dans le Dictionnaire de Théologie catholique, t. 13/2, col. 2902-2911, Paris, Letouzey et Ané, 1937 et H. ThURSTON, art. "Chapelet », dans le Dictionnaire d'archéologie chrétienne et de liturgie, t 3/1, col. 399-406, Paris, Letouzey et Ané, 1948.

${ }^{11}$ Miracles de Nostre Dame par Personnages XI (1339-1382), éd. G. PARIS et U. RoberT, t. 2, Paris, Firmin Didot, 1877 (Société des Anciens Textes français).

12 Alfonso X, el Sabio. Cantigas de Santa María, éd. W. Mettmann, Madrid, Castalia, 1986. Les Cantigas de Santa María ont été composées en galicien par un groupe de poètes d'origines très diverses réunis autour du roi Alphonse X le Sage entre 1252 et 1284. Elles alternent pièces narratives et pièces lyriques, pour un total de 427 poèmes. On lit une variante de ce récit dans les Laudes de Virgine Maria de Bonvesin Della Riva, éd. G. Contini, Poeti del Duecento, Milan, Ricciardi, 1960. 
Alors qu'il récite un jour ses prières dans une forêt, des voleurs s'approchent de lui, bien décider à lui dérober tous ses biens. Ils sont détournés de leur intention par une vision : il leur semble que Marie tend une rose à son dévot à chaque salutation angélique. Le geste de la sainte fige dans les esprits des hommes du XIII siècle la parfaite correspondance entre la prière et la fleur.

Le rosaire-dévotion comme le chapelet-objet correspondent donc à une série de cinquante, prières ou autres. Dans le cas du Rosarius, nous conservons les vestiges de deux séries. Si le nombre cent n'est pas pour surprendre dans un contexte marial (en leur premier état, les Cantigas de Santa María que nous citions ci-dessus comptaient cent poèmes), il n'est pas en parfaite adéquation avec la dévotion ici manifestement sous-jacente. Un autre miracle bien connu le montrera mieux qu'une analyse théologique, celui dont bénéficia la très dévote nonne Eulalie. Le texte latin largement répandu - et qui a servi de sources à la plupart des traducteurs romans de ce miracle - résume en ces termes les effets de l'intervention mariale :

Talem namque consuetudinem habebat quod sine ulla intermissione pro illius amore omni die «Ave Maria gratia plena, Dominus tecum» tot vicibus dicebat quot psalmos esse in Psalterio didiscerat. Et icirco ut istum numerum quaque die totum posset complere, videbatur illud angelicum «Ave» velocius quam deberet pronunciare. Sed nunc Matris Domini ammonicione roborata duas partes dimiserat, terciam cum magna diligentia amodo cantare morosius instituerat. ${ }^{13}$

De ce texte latin à ses traductions du XIII siècle, les données numériques restent étonnament stables ${ }^{14}$, ce qui atteste la pertinence de la comparaison au psautier davidique. Ainsi, selon toute vraisemblance, un troisième livre était prévu pour notre Rosarius, livre malheureusement aujourd'hui totalement perdu.

Voilà ce qu'on peut déduire des pratiques dévotionnelles. Mais le substantif « rosarius » est aussi connu pour avoir pu désigner des ouvrages didactiques ou scientifiques (le plus connu de la postérité étant le Rosarius d'Arnaud de Villeneuve). Or, notre compilateur est aussi un familier de ce type d'écrits,

${ }^{13}$ Ce texte latin correspond au $\mathrm{n}^{\circ} 521$ dans l'index d'A. PONCELET, « Initia miraculorum beatae Virginis Mariae », dans Analecta bollandiana, 22 (1902), pp. 241-360. Il est présent dans de très nombreux manuscrits latins. Nous le citons d'après Toulouse, BM, 482, f. 49r.

${ }^{14}$ Gautier de Coinci, Miracles de Nostre Dame, éd. F.V. KoENIG, Genève, Droz, 1961 (TLF, 95), II Mir 29, vv. 102-107 : «Toz ses salus la bone fame / Abriga luez dusqu'a cinquante, / Mais li myracles nos creante / Que cist cinquante mielz valurent / Et a la mere Dieu mielz plurent / Ne faisoient trois cinquantainnes. »; une autre traduction légèrement postérieure à celle de Gautier de Coinci se lit dans le manuscrit Paris, BnF, fr. 818, ff. 60rb-60va : «El avoit tel acotumance / Que senz nule entrelaisance (...) Tant 'Ave Maria' disit (...) / Quanz salmes avoit el sautier. (...) / Mais puis por l'amonestement / A la mere lo roi poissent / Les deus parties en laisa / Por ce que la tierce chanta / A reposees, doucemant.. » 
auxquels il emprunte largement pour les descriptions de choses. Il vaut dès lors la peine de rechercher les modèles « scientifiques » de notre compilateur.

L'un est donné par une comparaison des descriptions de choses avec celles que fournissent les prédécesseurs latins ; la source principale de notre Rosarius pour les premiers vers de chaque chapitre est Vincent de Beauvais, et son Speculum naturale. Curieusement, le Speculum historiale, qui contient plusieurs dizaines de miracles mariaux, n'a pas eu le même influence sur la rédaction de la compilation mariale.

Le second modèle à l'inverse est cité à plusieurs reprises comme inspirateur de récits miraculaires ; c'est Thomas de Cantimpré, un autre illustre dominicain. Il s'agit moins de l'auteur du Liber de natura rerum (qui ne semble pas avoir inspiré les descriptions de choses), que du rédacteur du Liber de apibus. C'est d'ailleurs cet ouvrage, composé vers 1250, qui est utilisé et parfois mentionné aux chapitres I, 26 («Le Livres des Aés nous devise / En tel maniere et en tel guise...», f. 10ra); I, 33 («Dont le Livre des Aés raconte / A ce propos un petit conte », f. 47vb) ; II, 6 ("Ou Livre des Aéz ai leü / Et moult volentiers l'ai veü... », f. 118va ); II, 18 (« Ou Livre des Aés a un conte... », f. 169vb) ; II, 24 ; II, 25. Il consiste en une longue description moralisée des abeilles, assortie de nombreux exempla. Son but est la glorification de la vie chrétienne et surtout la valorisation du culte marial. Si un modèle doit être trouvé pour notre Rosarius, on se doute que l'ouvrage de cet illustre prédécesseur dominicain, qui enchevêtre le commentaire de données naturalistes et narrations exemplaires, est un candidat parfait.

Toutefois, le Rosarius reste œuvre singulière, ne serait-ce que parce qu'elle fait disparaître les taxinomies habituelles. Le tableau en annexe donne la liste des «choses » traitées ici, selon leur ordre d'insertion dans le recueil. Pour le premier livre, nous conservons essentiellement l'herbier, où l'on repère des fleurs assez traditionnellement mariales (le rosier et la fleur de lis), d'autres dont l'usage christianisé ne surprend guère (l'olivier ou le senevé) et déjà quelques singularités (la menthe, le fenouil ou le persil)... N'oublions pas que Marie, mère de miséricorde, incomparable médecin, est invoquée pour guérir maints troubles somatiques ou psychiques. Ces plantes sont là pour nous vanter les mérites digestifs et purgatifs de la sainte Vierge. Tout aussi attendues sont la verge ou la fontaine, images traditionnelles de Marie dans l'eucologie ou la lyrique. Plus surprenante peut-être sera la panthère, dont le pelage multicolore évoque la multitude des vertus mariales. 
Nos repères se troublent tout à fait au second livre, pour lequel nous avons la chance de conserver un catalogue complet, donné après la table des chapitres. Nous sommes maintenant très loin du répertoire habituel des images mariales, dans lequel on peinerait à trouver mention du chameau, de la mandragore, de l'ortie ou de la malachite, pour n'attirer l'attention que sur quelques éléments. Par ailleurs, s'il existe peut-être un ordre dans la succession des chapitres, nous avons failli à le trouver, le passage du bestiaire au lapidaire ou à l'herbier ne semblant obéir à aucune logique thématique, numérique ni alphabétique. La seule constante est la légère dominante végétale, qui a permis à Gérard Gros de parler du «jardin des images mariales» à propos de ce manuscrit. Quant à l'inspiration, elle reste très singulière dans le paysage de la littérature mariale, ce qui n'est qu'une des raisons des possibles déroutes du lecteur moderne. Les considérations biologiques que recèle le manuscrit en sont un autre motif, tels les vers ci-dessous empruntés au chapitre 25 du premier livre :

Mirtus enpeche ne vomisse,

Grant aïde fait que je pisse ;

Mirtus toutes plaies raferme,

Et ou chief tient le chevol ferme. (Rosarius, f. 6vb)

Quoi qu'il en soit de nos méprises et surprises, le Rosarius est bien davantage qu'un simple jardin, c'est un univers à part entière ; le Rosaire est aussi Speculum mariale.

Si la trame reste mystérieuse, c'est que le compilateur n'a pas souhaité la mettre en évidence. Son œuvre est un tout, et s'il en fallait une preuve supplémentaire, l'existence même de prologues pourrait en apporter. Mais chaque chapitre jouit d'une très grande, sinon parfaite, autonomie. C'est à la cohésion interne de chacun d'eux que l'auteur apporte un soin particulier. D'un chapitre à l'autre, aucun renvoi, aucune allusion. Les redites sont nombreuses. Le Rosarius, ce n'est pas le rosier, mais la roseraie. Chaque chapitre est un arbuste indépendant dont l'éclat vient rehausser ou concurrencer celui de ses voisins. Chaque arbuste fait donc l'objet de soins spécifiques.

Pour faire croître un rosier, diverses méthodes peuvent être envisagées : la semence, quand la graine informe un nouveau végétal puis s'abolit en lui; la greffe, quand le greffon vient modifier l'essence du porte-greffe, les deux végétaux s'unissant pour ne plus faire qu'un; le marcottage, quand la tige enfouie dans la terre rejaillit un peu plus loin pour donner naissance à un arbre apparenté et pourtant autonome. Les trois modalités sont mises en œuvre dans le Rosarius. 
A défaut de pouvoir embrasser l'ensemble de la compilation, nous pouvons $\mathrm{du}$ moins arrêter notre regard sur quelques chapitres, afin d'y étudier l'application de ces différentes techniques. Commençons par le chapitre $45 \mathrm{du}$ second livre, consacré à la tortue.

D’une source qui reste à identifier, le Rosarius retient sept « qualités » pour la tortue, ordonnées comme suit : une carapace pour cacher sa chair; deux espèces, l'une marine, l'autre terrestre ; une apparence laide, voire effroyable ; des œufs plus petits que ceux des poules; quatre pieds; une tête de la taille de celle d'un serpent ; une chair dont la consommation guérit les faiblesses ou les fièvres. Le commentaire marial du premier point se devine sans difficulté : la carapace de la tortue est la métaphore de la façon dont la Vierge a su mettre sa chair à l'abri de toutes les tentations. La sainte est bien différente nous dit-on des débauchés de ce monde, qui se préparent un mauvais sort !

Sa char couvri de couverture

De abstinence, grief et dure;

C'est ce qui la fait nete et pure.

Mes mainte gent hui n'en ont cure :

Tuit quierent soulas et delis,

Roses, soucies, fleurs de lis.

Encor i a assez trop pis :

Desnuer son ventre et son pis.

Chierement leur sera vendu

Quant jugement sera rendu.

Les delices et les honneurs

Tourné leur seront lors a pleurs.

Je leur prie pour leur profist

Que veullent escouter cest dit. (Rosarius, ff. 255vb-256ra)

Ces deux derniers vers introduisent une chanson (RS 1507), exhortation à la conversion des jeunes gens, leur rappelant qu'ils ne connaissent pas l'heure de leur mort. Nous en citerons une strophe à titre d'exemple :

Plorez, plorez, bonne gent,

Plorez pour la grant destresse

Qui sera au jugement.

Forment vers vous s'adresse

Orgueil, luxure et ivresse,

Et tout li couvent

Vendront tuit a grant destresse

A leur dampnement.

Sage est qui se repent

Ainçois que vigne a tourment. (Rosarius, f. 256rb)

Le Rosarius contient ainsi une quinzaine de pièces musicales, avec leur notation, pour une dizaine d'entre elles relevées par Raynaud dans la 
Bibliographie des chansonniers francais ${ }^{15}$. Toutes sont accompagnées de la notation " quid" ». Pourtant, cees pièces ne sont en général connues que par le Rosarius, à l'exception de la chanson RS 1188 (chapitre 34 du premier livre), "Qui bien aime, a tart oublie $»^{16}$. La fiabilité du marquage des vers allogènes suffit à attester que notre Rosarius est manifestement le témoin d'un chansonnier pieux aujourd'hui perdu.

Continuons notre chapitre. Le retour au propos initial est simplement marqué par un vers : «A no tortue retournon». Suit le commentaire de chacune des qualités énumérées en tête de chapitre, selon un ordre rigoureux. Nous en délaisserons la majeure partie pour n'en relever que les derniers vers :

Tizique, ethique, pacient

Des quiex li cors vont a nient,

Segnefient adversitez

De chans, de mer et de citez;

Quar peril a par tout le monde.

Mes de ce la pucele monde

Garde et de mal'aventure,

Quant la personne prent en cure.

Presquez estoit anientez

Uns qui en mer fu tempestez.

Il requist la bele Marie;

A rrive l'arriva en vie.

De ce un conte especial

Est escrist ou Grant Marial. (Rosarius, f. 257vb)

Sans connaitre la teneur du récit qui suit, on constate que l'enchainement ne se fait que sur le pronom indéfini «nient» repris par «anientez ». Seule cette trame lexicale est exploitée, alors qu'une trame thématique en or s'offrait au compilateur. L'histoire est la suivante: des pélerins se rendant à Jérusalem doivent affronter une forte tempête, qui brise leur navire. Voyant le naufrage imminent, les prélats et autres notables se réfugient dans l'embarcation de secours, tout en exhortant le vulgum pecus à faire preuve de courage devant la mort. Un homme tombe à l'eau en voulant sauter dans la barque de secours ; miraculeusement, les rescapés le découvrent quelques jours plus tard sain et sauf sur le rivage. Il leur raconte y avoir été conduit par la Vierge. Entre la carapace de la tortue et l'embarcation miraculeuse fournie par le manteau de la Vierge, une analogie s'imposait que le conte populaire connait bien. Rien n'en est dit par le compilateur.

On ne peut pourtant penser que le lien entre les deux éléments essentiels du chapitre soit fortuit. C'est le cas dans tous les chapitres conservés. Rarement

15 Dans l'ordre d'insertion dans le Rosarius: 1963, 2114 (deux fois), 11, 1183, 1188, 2114, 458, 1551, 1177 ; G. RAYNAUD, Bibliographie des chansonniers français des XIII'e et XIVe siècles, Paris, Vieweg, 1884.

${ }^{16}$ Cette dernière est attestée par 3 autres témoins (Vatican, Bibl. apost., Reg. lat. 1490, chansonnier $\boldsymbol{a}$; Paris, BnF, naf. 1050, chansonnier $\boldsymbol{X}$; Paris, BnF, fr. 847, chansonnier $\boldsymbol{P}$. 
pourtant le Rosarius souligne cette cohérence. C'est tout à fait exceptionnellement qu'au chapitre 8 du second livre, après le miracle, le compilateur renoue avec la description de chose :

Celidoine donques est Marie

Qui enlumine et sauve vie.

Qui de bon cuer la vieut prier

A vile, a champ ou a moustier,

A nuli faillir ne saroit. (Rosarius, f. 126vb)

Revenons de notre côté au chapitre sur la tortue, et au récit du "Pèlerin sauvé de la noyade », qui met en pratique la technique du semis. Le compilateur n'est guère bavard lorsqu'il s'agit d'identifier la source de tel ou tel passage. Pour les descriptions de choses, aucun indice n'a été laissé. Pour les miracles, c'est avec un constant laconisme qu'il s'en réfère à Géraut de Frachet, Thomas de Cantimpré (voir supra) ou Étienne de Bourbon :

La Vie des Frerez nous recite

une narracion petite. (Rosarius, I, 23, f. 1va)

Ainsi la Vie le devise

Des Freres qui dist en tel guise... (Rosarius, I, 31, f. 33ra)

La Vie des Freres raconte,

Ou sont escrist plurieurs biau conte ... (Rosarius, II, 4, f. 109ra)

Uns devos moignes la requist,

Si com li Livres des Dons dist. (Rosarius, II, 1, f. 99ra)

Li Livre des Dons nous raconte,

Et Pierre de Clugni le conte... (Rosarius, II, 14, f. 154vb)

Dans tous les exemples ci-dessus, comme il ne peut y avoir d'ambiguité, les auteurs mentionnés ayant écrit en latin, le compilateur s'en tient à la formulation la plus simple et à la fois la plus ambiguë : c'est sa source latine qui est le sujet des verbes dire, conter, raconter. Le changement de langue semble visiblement une parade suffisante à toute incompréhension de l'auditoire. Il n'est pas indispensable de préciser davantage quel rapport entretient le texte du Rosarius avec ces auctoritates ${ }^{17}$.

Rien n'indique qu'il s'agit d'une traduction, ou plus exactement d'une réécriture, dont nous pouvons cerner les principales caractéristiques en rapprochant brièvement les versions françaises données pour ce miracle par notre compilateur et par son illustre prédécesseur Gautier de Coinci. Le texte

17 Je ne passerai pas en revue les nombreuses réserves de graines du Rosarius. Citons en bref complément la Legenda aurea de Jacopo da Varazze ou les recueils anonymes désignés par Mariale Magnum.. La plupart de ces sources ont été bien identifiées par A. LÅNGFORS, art. cit. 
latin utilisé par le Rosarius est facile à repérer, parce que le motif a fait l'objet de peu de variations, et que par ailleurs, le Rosarius nous cite comme source le « grant marial», c'est-à-dire l'une de ces grandes compilations de miracles latins de la Vierge dont les XIIe et XIII siècles ont été friands. Il ne peut que faire référence à la narration latine portant le numéro 442 dans le répertoire des miracles latins du père Poncelet ${ }^{18}$, et qui commence par «Erat navis in medio maris mediterranei peregrinis onusta ». Dans les Miracles de Nostre Dame, il apparait au chapitre 28 du second livre ${ }^{19}$.

Les entrées en matières respectives présentent peu d'écarts :

Erat navis in medio maris Mediterranei peregrinis onusta quorum devocio gracia oracionum parte Iherosolimitanas adhibat. (Poncelet 442)

Li livres dit ou leü l'ai

Qu'avint jadis que clerc et lai

En une nef la mer passoient ;

Au saint sepucre s'en aloyent. (Miracles de Nostre Dame, II, 28, vv. 7-10)

Jadis grant gent en mer estoient

Qui en Jherusalem aloient,

En cele precieuse terre

Que chascuns doit de cuer requerre

Pour l'amour du seignieur qui mort

I souffri pour vaincre le tort :

Ce fu Jhesus, le Filz Marie,

Qui la trahis fu par envie.

Nequedent tel mort n'avoit mie

Desservi ne tel vilennie. (Rosarius, f. 257vb)

Les deux auteurs français s'éloignent d'une traduction littérale, mais avec des motivations différentes: le second vers de Gautier n'a d'autre intérêt que métrique (le poète utilise fréquemment comme cheville métrique le syntagme « et clerc et lai»). En revanche, le Rosarius offre ici une brève contemplation du mystère de la Passion qui tranche sur le ton purement informatif des deux autres textes. Avant d'aller plus avant dans son récit, il propose à son auditoire de méditer quelques instants sur le scandale de la Croix. Écriture bien différente de celle du poète soissonnais, écriture qui préfère la sobriété aux effets dramatiques, très soulignés chez Gautier dans le premier épisode, celui du naufrage :

18 A. PONCELET, art. cit. Nous citons le miracle latin d'après l'édition qu'en a donnée B. DUTTON, Gonzalo de Berceo. Obras completas II. Los Milagros de Nuestra Señora, Londres, Tamesis Books, 1971, pp. 183-184)

${ }^{19}$ De nombreux poètes romans ont utilisé ce texte latin. On pourra en lire des traductions du XIII siècle dans la collection lyonnaise du manuscrit Paris, BnF, fr. 818, ff. 25vb-27va, dans les Milagros de Nuestra Señora de Gonzalo de Berceo, nXXII (éd. B. DuTTON, op. cit.), dans les Cantigas de Santa María d'Alphonse X, n ${ }^{\circ} 23$ (éd. W. METTMANN, op. cit.), dans la collection en prose provençale, $\mathrm{n}^{\circ} 10$ (éd. J. UlRICH, « Miracles de Notre Dame en provençal », dans Romania, 8 (1879), pp. 12-28. 
Quant li maistres de la nef vit

Que la mors a l'uel li pendoit

A demi pié, voire, a plain doit,

Entrez s'en est isnelement,

Et uns evesques ensement

Et li plus haut qui la estoyent,

En une barge qu'il avoient.

Mais uns de cialz qui en la barge

Cuida saillir de la nef large

En mer chaï, et mers l'englot

Tout ausi tost com un seglot. (Miracles de Nostre Dame, II, 28, vv. 18-28)

Ces vers ne sont guère plus longs que le passage latin qui les a inspirés ${ }^{20}$, mais Gautier y joue des effets de crescendo, d'un danger qui «a l'uel li pendoit», ce qui le tient encore à quelque distance, jusqu’à un danger qui touche l'homme «a demi pié », et en moins d'un vers " a plain doit », le rythme de la phrase adoptant celui de la noyade et de ses hoquets, du flot qui emporte tout avec lui (qu'on en juge par les coordinations). L'auteur du Rosarius s'en tiendra lui à une traduction sensiblement abrégée de son modèle :

Li evesques et li maronnier

Le peril weullent eschiver :

En la cymbe sont descendu;

Par ce de mort sont deffendu.

Uns hons qui en hate i sailloit

Et qui pou avisez estoit,

Dedens la mer chiet et trebuche. (Rosarius, f. 258ra)

Pareil contraste entre les deux traducteurs français se lit dans le récit fait par le miraculé à la fin du miracle :

Il respondi que quant cheoit

La vierge Marie apeloit

Et bien l'avoit en son memoire.

Que fist la mere au roy de gloire?

«En la mer me prist, ou parfont,

Ou perdu sont cilz qui la vont.

De son biau mantel m'a couv[ert],

Je ne scey s'estoit gris ou ver[t];

Sain et sauf m'a cy amené. » (Rosarius, f. 258rb) ${ }^{21}$

20 «Festinus igitur cimbam que magnarum navium more intus ferebatur navi exponit, in mare deponit et cum episcopo quodam qui inter ceteros aderat, et aliis quibusdam nobilioribus in eam descendit. Unus tamen, cum a magna in parvam vellet descendere navem, in mare decidit pelagique statim mersus ad ima nusquam comparuit. » (Poncelet 442)

21 «Quid socii, inquit, sic me admiramini salvatum quem salvare dignata est per quam salus venit omnium ? Beate quippe Dei genitricis nomen Marie, cum in aqua eadem caderem, exclamavi et sic eius et memoriam habendo et nomen inclamando ad ima pelagi deveni. At ipsa misericordie mater que memorum suorum immemor esse nullatenus potest, haut mora mihi sub undis astitit pallio me suo clementer contexit, et contectum sub aquis litus adusque perduxit. » (Poncelet 442) 
À ces neuf vers du Rosarius en correspondent quatre-ving-six (vv. 121-206) chez son prédécesseur. S'il y a convergence entre les deux poètes, c'est dans la volonté de développer une proposition du texte latin, «et ut sua peccata confidentes suas devote animas Deo commendarent admonuit». Tous deux prédicateurs, ils appuient cet appel à la confession : Gautier de Coinci en jouant ironiquement de l'opposition entre les confès silencieux dont les âmes s'envolent vers le ciel sous forme de colombes et le prolixe évêque regrettant amèrement d'avoir mis son corps en sûreté après avoir exhorté autrui à une mort courageuse (vv. 35-92) ; le Rosarius en glosant le psaume 33

$\mathrm{N}^{\prime}$ i a remede ne confort

Mes que souffrir, et en pacience,

Et avoir nete conscience.

Asseür muert cilz qui l'a nete,

Non celui qui l'a deshonneste.

Ps. Mors peccatorum pessima,

Pour ce descendent ad yma;

«Tres pesme est la mort du pecheur»,

A grieté muert et a douleur.

Descent a douleur doulereuse ;

Il n'en est nule plus crueuse ! (Rosarius, v. 258ra)

Le compilateur du Rosarius, tout autant - et peut-être plus - que Gautier de Coinci, ne garde que la substantifique moelle de son modèle, et fait véritablement sien le récit, ce qui n'est pas le cas d'autres traductions romanes du même récit. Comme Gautier, il n'hésite pas à gloser, développer, mais dans une perspective sensiblement différente: la contemplation mystique reste sa préoccupation première, bien loin devant la dramatisation. Non que cette dernière soit absente : elle vient agréablement égayer les récits les plus longs (voir le chapitre 26 du premier livre) ; mais notre poète anonyme lui préfère la rumination des saints préceptes.

Finissons-en avec le chapitre sur la tortue. Après le miracle vient une transition un peu abrupte, avant que le compilateur ne cède la parole à la Vie des Pères22.

Ta purté, se tu as perdue,

Par penance sera rendue.

Se en ton cors la vieus porter,

Ele sieut bon fruit aporter.

Penance donc en ton cors porte

Se tu vieus entrer en la porte

De paradis, ou n'entre point

Qui par penance ne se point.

22 Conte XLIII, «De l'ermite qui sala son pain », éd. F. LECOY, La Vie des Pères, t. III, Paris, Picard, 1999 (Société des Anciens Textes français). 
Dire vous weil un examplaire

Pour atraire vous a bien faire,

Especiaument a penance.

Temps est d'amesgroier sa pance,

Et qui a eu les soulas

Maintenant die « Helas! Helas!»

Chier leur sera le temps vendu

Que folement ont despendu! (Rosarius, f. 258rb)

Dans le miracle il n'était nullement question ni de pureté ni de pénitence. C'est à la fin du commentaire de la première qualité de la tortue qu'il faut aller chercher l'amorce de ce passage :

Penance face le pecheur

Se monter vieut a celle honneur

Ou nus ne monte se penance

Ne fait et aplatit sa pance.

Cy aprés en orrez un dit

D'un qui grief penance fist. (Rosarius, f. 256vb)

Ce dernier élément du chapitre nous conduit à évoquer une deuxième technique horticole, le marcottage. Le texte du Rosarius semble brusquement plonger une racine dans le sol, qui rejaillit sous la forme d'un arbuste parfaitement autonome quelques vers plus loin. Dans tous les cas, il s'agit alors non de pièces de la plume du Rosarius, mais d'emprunts.

Revenons au premier livre du recueil, au chapitre 35 consacré au baume, pour observer de plus près cette pratique. La rubrique annonce deux éléments uniquement, un miracle, et un « ditié » ${ }^{23}$. Sur l'enchaînement de la description et du miracle, on pourra faire le même constat que précédemment, puisque c'est de nouveau sur un jeu verbal plus que sur les potentialités de la chose décrite que s'amorce le miracle :

Molt est dame de grant confort

Especiaument a la mort :

A la mort ses amis conforte.

Ele est de paradis la porte.

De son confort bien nous raconte

La vie des freres, qui ainsi conte. (Rosarius, f. 58ra)

La rime confort / mort offre ici la possibilité de jouer sur un autre motif courant de l'hagiographie mariale, celui de la Mère de Miséricorde. À l'heure de sa mort, un dévot exemplaire reçoit la visite de Marie, qui l'assure de son aide au moment du grand passage. Le thème initial du chapitre, le «baume », n’est

23 «De Nostre Dame qui visita frere Pierre a sa mort. Item, un ditié de la vertu et puissance de la mort » (Rosarius, f. 57ra) 
nullement reconvoqué ici pour appuyer la notion de réconfort. On sent pourtant qu'il est sous-jacent.

Le miracle commence donc par le portrait d'un moine exemplaire, portrait sur lequel le compilateur greffe un long sermon sur les bons et les mauvais moines. L'attaque contre la bonne chair débouche sur :

Moigne notablement se note

Quant, pour boire vin, vent sa cote.

Il puet bien jurer par sa bote:

«Sot sui» (se fame est, c'est « sote»)!

Sachies pour voir que moigne ivroigne

Ne fera ja bonne besoigne. (...)

$\mathrm{Li}$ moignes qui a guersay boet

Fort est que en paradis voit. (Rosarius, ff. 58ra-rb)

Après quelques vers, l'expression "boire a guersay ", à laquelle le lecteur n'aura peut-être pas prêté grande attention, rejaillit comme racine d'un dit satirique, transmis également par le très fameux manuscrit Paris, BnF, fr. 837. Le cas n'est pas isolé, puisque les deux manuscrits ont en commun une dizaine de textes (voir tableau 2).

S’il s'agit bien du même Dit de Guersay dans les deux manuscrits, chaque copie présente un incipit propre :

Mon cuer si m'a doné matere

Et me semont que je m'atere

Et qu'a un ditié fere essai.

Cele qui est et virge et mere

Qui porta son fil et son pere... (Paris, BnF, fr. 837, f. 237vb)

Reson m'esmuet que je ceus fiere

Qui de boire ont gloute maniere.

Contre tiex gent un dit feray.

Cele qui est et fille et mere

Qui porta son filz et son pere...(Rosarius, f. 58rb)

Le Rosarius intervient sur des vers à la première personne. De façon comparable, il omet la première strophe du Doctrinal sauvage :

Or escoutez seignour que Diex vous beneie

S'oirez bons moz noviaus qui sont sanz vilonie

Ce est de Doctrinal qui enseigne et chastie

Le siecle qu'il se gart d'orgueil et de folie. (Paris, BnF, fr. 837, f. 335va)

Il délaisse également les deux premières strophes du Regret Nostre Dame d'Huon le Roi de Cambrai où ce dernier explique son projet. Bref, il n'insère que les voix qu'il peut faire totalement siennes, substituant des vers personnels à ceux écrits à la première personne par ses prédécesseurs. Le résultat est une compilation polymorphe, mais non polyphonique. 
Si les points de rencontre sont nombreux, il importe de souligner qu'il n'y a pas d'influence directe du fr. 837 sur le Rosarius. Les leçons de celui-ci sont souvent différentes de celles de celui-là. Pour les textes à ample tradition, et l'on pense en particulier aux Vers de la mort, les deux recueils divergent fortement ${ }^{24}$. Il y a toutefois là une familiarité de contenu qui intrigue, d'autant que le Rosarius et le fr. 837 proposent parfois des textes différents sur des thèmes proches et rares à la fois. Ainsi de la Patenôtre de l'usurier ou du Dit des moustiers de Paris.

La formule employée par le compilateur pour insérer le Dit de Guersay requiert également l'attention :

Un ditié contre les gloutons

En nostre livre ainsi entons.

Qui le ditié fist je ne say

Mes mult reprent boire au guersay.

De trop boire entends que dist

Ciz qui le ditié tel en fist. (Rosarius, f. 58rb)

L'emploi du verbe enter pour signifier l'insertion dans une œuvre de pièces allogènes, sans être unique, n'est pas très courant. Dans le Rosarius, il est employé une autre fois dans la compilation au chapitre 27 du premier livre, avant l'insertion d'une paraphrase du Pater

En un livre je l'ai trouvee

Escripte et en mon uevre entee. (Rosarius, f. 15rb)

Sans en exagérer la portée, puisque dans aucun des deux cas la métaphore végétale n'est développée, et qu'ailleurs le compilateur emploiera simplement le verbe mettre, on y verra tout de même un jeu avec le titre de l'œuvre mariale. Sur tel ou tel rosier de son jardin, le compilateur greffe les paroles d'autrui pour donner plus d'agrément et d'ampleur aux siennes.

Avec ce dit satirique, nous ne sommes pas parvenus à la fin du chapitre. La reprise de la narration après le dit de Guersai se fait de nouveau par une brève formule («Or retournon a frere Pierre, / Qui par tens sera mis en terre. »), puis le miracle se conclut sur la nécessité de servir la Vierge. De ce service dévot, le compilateur donne immédiatement l'exemple en insérant la chanson RS 1183. Mais elle ne saurait faire oublier que nous avions vu plonger en terre une autre branche, le thème de la mort, thème central du miracle. Après la chanson, cette branche donne naissance à un nouveau surgeon :

${ }^{24}$ Le Rosarius présente un texte plus proche de celui établi par F. WULFF et E. WALBERG, Paris, Firmin Didot, 1905 (Société des Anciens Textes français) que le fr. 837. 
Pour ce que j'ai fait mencion

De mort qui a corrupcion

Tout met, un ditié en escript

$[\mathrm{m}]$ is ai. Je ne sai qui le fist...

Il est pourfitable, ce cuit.

Or l'escoutes, ne vous ennuit! (Rosarius, f. 60va)

Le texte d'Hélinant est alors donné en son entier, le compilateur abondant dans le sens de son prédécesseur pour appeler les cœurs à la conversion. Le chapitre pourrait s'arrêter là, c'est d'ailleurs ce que laissait entendre la rubrique. Mais une branche un peu sauvage s'en échappe :

S'ainsi vis continuelment,

Tu mourras plus legierement.

De mourir ne t'esbahi mie !

Moru Jhesus li fix Marie.

Sa mere en fu moult esmarie;

[Or entends] comme pleure et crie. (Rosarius, f. 63ra)

Le premier couplet d'octosyllabes fournirait une très bonne conclusion aux Vers de la Mort. Mais le compilateur se souvient sans doute des craintes du mourant dans d'autres versions du miracle qu'il vient de raconter - et peut-être dans sa source. L'évocation de la mort du Christ nous rappelle que nous sommes appelés par là à la Vie, ce qui n'enlève rien à l'angoisse de celui qui franchit le passage, ni à la peine de ceux qui restent. La plus sainte des femmes elle-même n'y a pas échappé ; c'est elle dont nous écoutons alors la Plainte. Le compilateur reprendra la parole après la Plainte de la Vierge, pour quatre vers de conclusion il faut bien l'avouer assez fades, mais qui révèlent le souci de clore chaque unité, souci très affirmé dans le premier livre, moins achevé dans le second :

Mout eut la dame grant douleur!

Nous li prirons par sa douceur

Que, si penson a la Dieu mort,

Qu'arriver puissons a bon port. Amen (Rosarius, f. 64rb)

Penchons-nous pour finir sur le chapitre le plus long conservé, avec plus de 1700 vers. On ne s'étonnera guère qu'il s'agisse de celui sur le rosier... Voici quelle en est la rubrique :

De la pucele angroissie qui se feri ou ventre d'un coutel que Nostre Dame envoia a m[estre] Jourdain, et puis devint nonain. Item un dit de la rose. Item la contenance aus dames. xxxi (Rosarius, f. 32rb)

Outre les citations attendues de l'Ancien Testament annonçant la Vierge sans épines, le Rosarius évoque les vertus médicinales de la rose. Dans le miracle, comme nous l'avons vu précédemment, le compilateur insère des exhortations morales. La première arrive très vite, après à peine 40 vers de narration : 
De dire $1 a^{25}$ n'aies pas honte;

En tans, en lieu di la de plain,

Ne la muce pas en ton sain

[S] i comme font molt de flateur

Qui de la dire ont grant peur.

Qui a court est se garde bien

Que de flater ne sache rien:

Flateur ne plaisent grain a Dieu;

Il tiennent a court mauvés lieu. (Rosarius, f. 34va)

Comme nous l'avons vu faire auparavant, le Rosarius rebondit sur le substantif «flateur » pour insérer des propos allogènes :

Pou est qui de court weillent estre apostate:

Je ne m'en merveil pas, car chascun les i flate

Ou il flatent autrui pour qu'en ne les surbate,

Car meine sa cuisse ${ }^{26}$, qui un petit la grate.

Court sieurre et non flater a homme mendiant

Vient de trop grant vertu, selonc mon escïant;

Je ne sai si preudomme deci en orient

Qui un pou en ce cas ne se voist ortiant ${ }^{27}$.

Mauvais homs li oncles estoit,

Qui sa niece ainsi conseiloit,

Quar aprés le fait de luxure

Conseile ocirre sa porteure. (ibid.)

La situation est pourtant ici sensiblement différente des cas évoqués précédemment. En effet, cette citation ne conserve pas son autonomie: le changement de mètre est bien évidemment sensible, mais on remarquera qu'à la reprise, il n'y a aucun effort de transition du compilateur, tout simplement parce qu'il n'a pas senti de solution de continuité.

Ce n'est plus à du marcottage que se livre ici le poète, mais bien à une greffe : les paroles d'autrui fusionnent avec les siennes pour ne plus faire qu'un. Ce traitement bien particulier concerne exclusivement les textes didactiques, les paroles de prédication. C'est la méthode employée par exemple au chapitre 29 du premier livre une trentaine de vers du sermon De la doutance de la mort de Gautier de Coinci. C'est celle utilisée ici pour des vers extraits du Testament de Jean de Meun.

Le Rosarius semble avoir souhaité intégrer de la sorte l'ensemble du Testament, mais en en redistribuant les strophes. Il reste difficile de se faire une idée juste de la répartition et des quelques omissions en raison des lacunes matérielles, mais nous avons tenté de rendre visible par le tableau 3 l'usage fait

\footnotetext{
25 Il est question de la vérité.

${ }^{26}$ Dans les autres témoins : mainsnie s'acoise.

27 Dans les autres témoins : ornant.
} 
de ce long texte (rappelons que dans l'édition qu'en a donnée Silvia Buzzetti Gallarati28, le Testament compte 530 quatrains, soit 2120 vers alexandrins). Certains passages semblent toutefois pouvoir avoir été omis «à dessein». Comme pour certains textes présents dans le fr. 837, le Rosarius a certainement omis les strophes initiales et finales, d'une tonalité très personnelle. Ils délaissent également les vv 1797-1804, vers de transition (« Or avez vous oy les noms et la nature / Des sept pechiez mortels et de leur norreture »).

La lacune considérable des vv. 849-1168 pourrait bien de même être un effet de la volonté du compilateur: Jean de Meun s'y livre à des attaques assez virulentes contre les ordres mendiants, capteurs de testaments. Or, si le compilateur du Rosarius reste anonyme, Langfors avait épinglé plusieurs indices «autobiographiques» dans la compilation permettant de penser à un prédicateur dominicain. Bien plus, les miracles de la Vierge mettant en scène Dominique ou Jourdain de Blaye sont anormalement nombreux dans le recueil. L'omission des passages « anti-dominicains » de Jean de Meun apporte de l'eau à ce moulin.

Là où sans doute Langfors s'est partiellement trompé, c'est en arguant d'une bonne connaissance de la région soissonnaise pour faire du Rosarius une œuvre soissonnaise, héritière de Gautier de Coinci. Le poète du XIIIe siècle occupe une place très mineure (40 vers dans un peu plus de 40000 conservés). Inversement, des auteurs très parisiens occupent une place de choix dans la compilation : on pense à Jean de Meun, ou à Rutebeuf. Le Dit des Moustiers de Paris témoigne de la même familiarité avec la ville universitaire et royale. Qui qu'il fût, le compilateur avait par ailleurs à sa disposition une bibliothèque spirituelle et profane exceptionnelle.

Quant au manuscrit, et nous ne nous prononcerons pas ici fermement sur le fait qu'il s'agisse ou non d'un manuscrit d'auteur, il sort d'un atelier parisien, et ne saurait être beaucoup plus tardif que la compilation. Enfin, il porte l'ex-libris du couvent des dominicains de Poissy. Si l'on veut bien reconsidérer un instant l'histoire de l'abbaye royale, on renoncera sans doute à l'hypothèse de Langfors. La charte de fondation de l'abbaye, en 1304, prévoit la présence de prédicateurs dominicains auprès de la prestigieuse communauté féminine, prédicateurs en poste pour quelques années seulement, très liés au couvent de la rue SaintJacques à Paris.

Ne pourrait-on avoir ici affaire à l'un d'entre eux ? Un prédicateur dont la vaste culture livresque couvrirait aussi bien les encyclopédies et autres ouvrages scientifiques que la lyrique mariale ou la littérature profane en ses tendances

28 S. BuZZetTi-Gallarati, Le testament Maistre Jehan de Meun : un caso letterario, Alessandria, Edizioni dell'Orso, 1989. 
satiriques. Le public visé par le prédicateur est vaste : tout homme et toute femme désireuse de faire son salut. Mais plus ponctuellement, comme dans le chapitre sur le baume que nous avons cité, l'auteur s'adresse visiblement à une communauté de femmes :

De ce pri cele ne soies mie quar tele ne fu pas Marie.

C'est la fourme de ta vie ;

Abeesse est de t'abaie.

Vif si comme vit t'abeesse ;

A sa vie la tieue adresse. (Rosarius, f. $57 \mathrm{va}$ )

Qu'on ne s'y méprenne pas pourtant. Ces textes ne sont pas des sermons. Ce sont des textes à dire ou à lire et à chanter, mais ni leur longueur ni leur style ne se prêtent à une lecture en chaire. Leur autonomie les rend aptes à une lecture ponctuelle, et le destinataire en est alors l'âme chrétienne. Leur mise en recueil en fait une œuvre dévotionnelle de grande ampleur, dont le destinataire est la Vierge. Si chaque rosier fait l'objet de soins horticoles minutieux, c'est pour la sainte que le miroir se fait roseraie. 
Tableau 1

\begin{tabular}{|c|c|c|c|}
\hline & & Menthe $(I, 24)$ & \\
\hline & & Myrte $(I, 25)^{29}$ & \\
\hline & & Fenouil $(\mathrm{I}, 26)$ & \\
\hline & & Sénevé (I, 28) & \\
\hline & & Corail $(I, 29)$ & \\
\hline \multicolumn{4}{|l|}{ Panthère (I, 3O) } \\
\hline & & Rosier (I, 31) & \\
\hline & & Cannelle (I, 32) & \\
\hline & & Olivier (I, 33) & \\
\hline & & Fleur de lis $(\mathrm{I}, 34)$ & \\
\hline & & Baume (I, 35) & \\
\hline & & Persil $(\mathrm{I}, 36)$ & \\
\hline & & Amandier (I, 37) & \\
\hline & & & Fontaine $(\mathrm{I}, 38)$ \\
\hline & & Plantain $(\mathrm{I}, 45)$ & \\
\hline & & & Verge $(I, 50)$ \\
\hline & & & Château (II, 1) \\
\hline \multicolumn{4}{|l|}{ Hirondelle (II, 2) } \\
\hline & Kabratés (II, 3) 30 & & \\
\hline & & Armoise (II, 4) & \\
\hline & & Laurier (II, 5) & \\
\hline \multicolumn{4}{|l|}{ Cigogne (II, 6) } \\
\hline & & Mandragore (II, 7) & \\
\hline & & Chélidoine (II, 8) & \\
\hline & & & Galaxie (II, 9) \\
\hline \multicolumn{4}{|l|}{ Brebis (II, 10) } \\
\hline & & & Anneau (II, 11) \\
\hline
\end{tabular}

30 « sorte de pierre » pour les éditeurs, « kabrates » chez Vincent de B 


\begin{tabular}{|c|c|c|c|}
\hline & & & Dé (II, 12) \\
\hline & & «Serpentine» (II, 13) & \\
\hline & & Oignon (II, 14) & \\
\hline \multicolumn{4}{|l|}{ Baleine (II, 15) } \\
\hline \multicolumn{4}{|l|}{ Caladrius (II, 16) } \\
\hline & Galactite (II, 17) & & \\
\hline \multicolumn{4}{|l|}{ Salamandre (II, 18) } \\
\hline & & & Lait (II, 19) \\
\hline & & Safran (II, 20) & \\
\hline \multicolumn{4}{|l|}{ Abeille (II, 21) } \\
\hline & & Galbanum (II, 22) & \\
\hline & & & Ange (II, 23) \\
\hline & & Laitue (II, 25) & \\
\hline & & Mauve (II, 26) & \\
\hline & & Pin (II, 27) & \\
\hline & Malachite (II, 28) & & \\
\hline & & Mûrier (II, 29) & \\
\hline & & & Terre (II, 30) \\
\hline \multicolumn{4}{|l|}{ Cygne (II, 31) } \\
\hline & & Mandegloire (II, 32) & \\
\hline & & & Soleil (II, 33) \\
\hline & & & Soleil (II, 34) \\
\hline & & & Fontaine (II, 35) \\
\hline \multicolumn{4}{|l|}{ Castor (II, 36) } \\
\hline & & Hellébore (II, 37) & \\
\hline & & Lierre (II, 38) & \\
\hline & & & Etoile polaire (II, 39) \\
\hline & & Aneth (II, 40) & \\
\hline
\end{tabular}




\begin{tabular}{|l|l|l|l|}
\hline & & & Ciel (II, 41) \\
\hline Rossignol (II, 42) & & & \\
\hline & & & Vierge (II, 43) \\
\hline Colombe (II, 44) & & & \\
\hline Tortue (II, 45) & & & \\
\hline Chameau (II, 46) & & & \\
\hline Faucon (II, 47) & & & \\
\hline & & *Violette (II, 48) & \\
\hline & & *Ortie (II, 49) & \\
\hline & & *Palme (II, 50) & \\
\hline
\end{tabular}

Tableau 2

\begin{tabular}{|l|l|l|}
\hline Paris, BnF, fr. 837 & & Chapitre du Rosarius \\
\hline 70va-73va & Vers de la mort, d'Hélinant de Froidmont & I, 35 \\
\hline 93rb-95vb & Regret Notre Dame d'Huon le Roi & II, 19 \\
\hline $112 \mathrm{rb}-114 \mathrm{rb}$ & Quinze signes du Jugement & II, 13 \\
\hline 179rb-180rb & Neuf joies de Rutebeuf & II, 1 \\
\hline 186ra-187ra & ABC Plantefolie & I, 33 \\
\hline 237vb-238va & Dit de Guersai & I, 35 \\
\hline 274ra-rb & Patenôtre farcie & I, 25 \\
\hline 328a-328vb & Ave Maria de Rutebeuf & II, 21 \\
\hline 334ra-335va & Doctrinal Sauvage & II, 9 \\
\hline 335va-336vb & Chantepleure & II, 32 \\
\hline
\end{tabular}




\section{Tableau 3}

Dans la colonne de gauche, les numéros portés en gras signalent les quatrains copiés deux fois dans le Rosarius. Dans la colonne de droite, les cases hachurées correspondent aux lacunes liées selon toute vraisemblance à l'état matériel du manuscrit; les cases grisées aux lacunes relevant de la volonté du compilateur.

\begin{tabular}{|c|c|}
\hline $\mathrm{N}^{\circ}$ des vers dans l'édition Buzzetti & Insertion dans le Rosarius \\
\hline \multicolumn{2}{|l|}{$1-8$} \\
\hline $9-24$ & 4 \\
\hline \multicolumn{2}{|l|}{$25-36$} \\
\hline $37-64$ & 11 \\
\hline \multicolumn{2}{|l|}{ 65-68 } \\
\hline $69-92$ & 3 \\
\hline $93-100$ & 33 \\
\hline $101-112$ & 35 \\
\hline \multicolumn{2}{|l|}{ 113-132 } \\
\hline 133-144 & 5 \\
\hline $145-240$ & 2 \\
\hline $241-280$ & 22 \\
\hline $277-304$ & 8 \\
\hline \multicolumn{2}{|l|}{ 305-308 } \\
\hline $309-404$ & 12 \\
\hline $405-440$ & 24 \\
\hline $437-496$ & 9 \\
\hline \multicolumn{2}{|l|}{ 497-512 } \\
\hline $513-520$ & 14 \\
\hline $521-556$ & 26 \\
\hline $557-592$ & 17 \\
\hline \multicolumn{2}{|l|}{ 593-596 } \\
\hline $597-644$ & 16 \\
\hline $645-672$ & 15 \\
\hline
\end{tabular}




\begin{tabular}{|c|c|}
\hline 673-696 & \\
\hline $697-716$ & 27 \\
\hline $717-732$ & \\
\hline $733-784$ & 13 \\
\hline $785-840$ & \\
\hline $841-848$ & 18 \\
\hline $849-1168$ & \\
\hline $1169-1212$ & 28 \\
\hline $1213-1255$ & 36 \\
\hline $1257-1348$ & \\
\hline $1349-1356$ & 34 \\
\hline $1357-1360$ & \\
\hline 1361-1432 & 25 \\
\hline $1429-1468$ & 29 \\
\hline 1469-1556 & 19 \\
\hline $1561-1640$ & 32 \\
\hline $1637-1648$ & 30 \\
\hline $1649-1680$ & 10 \\
\hline 1681-1984 & \\
\hline $1685-1736$ & 20 \\
\hline 1733-1796 & 31 \\
\hline $1797-1804$ & \\
\hline $1805-1836$ & 21 \\
\hline 1837-1972 & 23 \\
\hline $1973-2024$ & 1 \\
\hline 2025-2048 (+2049-2056 biffés) & 6 \\
\hline 2049-2096 & 7 \\
\hline $2096-2120$ & \\
\hline
\end{tabular}

